\title{
Targeting ncRNAs to combat metastases
}

"The advent of high-throughput techniques for the study of the whole miRNome (defined as the full spectrum of miRNAs in a genome) in one biologic sample, has allowed the detection of specific signatures of deregulated miRNAs in cancer versus normal tissues, with diagnostic and sometime prognostic implications."

\section{KEYWORDS: cancer $\approx$ metastases $\approx$ miRNAs $\approx$ ncRNAs $\approx$ oncogene $\approx$ tumor suppressor gene}

Metastases are the main cause of cancer-related deaths [1]. Metastasis formation is a multistep process that involves neoplastic cells dissociating from the primary tumor, invading surrounding tissues, entering the circulatory system (lymphatics and/or blood vessels), surviving during transport until they reach their site of metastases, and then recapitulating the same processes in reverse (exit the circulation, invade the new site stroma, and proliferate at the ectopic site in response to local growth factors) [2]. Metastasization is an extremely inefficient process, since it has been estimated that of the approximately 4 million cells entering the vascular system per gram of tumor per day, less than $0.01 \%$, actually develop distant metastases [2]. Fortunately, for cancer patients, this inefficiency is probably due to the fact that each step in the metastatic cascade of events is rate-limiting and requires a specific coordinated expression of genes and spatiotemporal expression of proteins.

The term ncRNA includes a group of RNAs that are not translated into proteins, with several functions in the cell [3]. Traditionally considered as by-products of 'junk' DNA genes, they have recently come into the limelight as the main regulators of gene expression, therefore catching the attention of a vast group of scientists. Currently, miRNAs are the most extensively studied group of ncRNAs. They are small ncRNAs, 20-24 nucleotide long, which regulate gene expression by binding mainly (but not exclusively) to the $3^{\prime}$-UTR region of their target mRNAs [4]. The first connection between cancer and miRNAs was based on the discovery that miRNAs are located in cancer-associated genomic regions [5], and that they are frequently deregulated in human tumors [6-8]. Overall, research in the miRNA field has focused on three main topics, which are somewhat temporally sequential. The advent of high-throughput techniques for the study of the whole miRNome (defined as the full spectrum of miRNAs in a genome) in one biologic sample [9-11], has allowed the detection of specific signatures of deregulated miRNAs in cancer versus normal tissues, with diagnostic and sometime prognostic implications [12-14]. After this first topic, miRNA research has focused on the more systematic detection of miRNA target genes, in an attempt to understand the pathogenetic implications of the observed cancer-specific aberrations of the miRNome [15,16]. Finally, researchers have started to understand that each miRNA does not have one target, but several targets, and have focused on trying to determine whether a single miRNA modulates several target genes involved in a common pathway [17]. All three of these phases of miRNA research (Phase I: highthroughput miRNA detection; Phase II: identification of miRNA target gene(s); and Phase III: pathway miRNAs) have one aspect in common: they investigate miRNA function inside the cell. In 2008, Tavazoie et al. published the first report that miRNAs are able to suppress breast cancer metastasis [18]. This work represents a milestone in miRNA (and ncRNA) research, not only since it detects some of the miRNAs modulating the most lethal aspect of malignancy, but also because it moves the spotlight from the 'intracellular domain' to the extracellular effects of miRNA deregulation in cancer. Subsequently, several other miRNAs have been discovered, able to either suppress or promote metastasis formation: the so-called 'metastamirs' (for an excellent review see [19]).

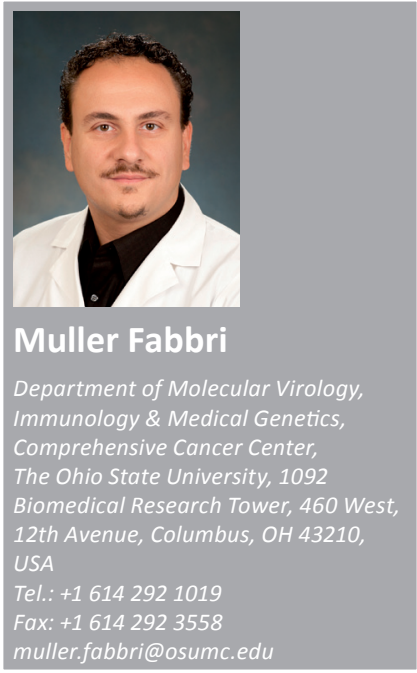

future medicine $^{\text {piss }}$ fs 
The interest in miRNAs involved in the genesis of cancer metastases, moved the attention of researchers from the intracellular to the extracellular environment and stimulated new research avenues. One of these was the detection of miRNAs outside the cells, in human fluids such as blood, as biomarkers to diagnose cancer $[20,21]$. Interestingly, it has also been shown that miRNAs circulate enclosed in tumor-derived exosomes of endocytic origin [22], an observation that provides the rationale for a possible cell-to-cell transfer of miRNAs, and therefore, of a possible intercellular cross-talk in an 'endocrine-like' fashion. This hypothesis is extremely intriguing, although more studies are needed to fully support it. Nonetheless, both the discovery of metastasis-specific miRNAs and of circulating miRNAs ultimately point towards an involvement of ncRNAs in the metastatic process. Recently, Kan et al. have published an extensive study on the macromolecules (proteins and ncRNAs) modulating invasiveness in cells of various cancers [23]. The authors found 28 miRNAs and one long ncRNA implicated in cell invasiveness, which represents the first key step for successful metastasization. Moreover, another ncRNA (HOTAIR long ncRNA) is able to promote cancer metastasis by inducing epigenetic changes in the chromatin state of cancer cells [24]. Therefore, ncRNAs emerge as increasingly attractive targets for anticancer treatment. However, the development of new ncRNA-based therapeutic tools to combat metastases must take into consideration several issues before they can be properly designed for clinical trials. First, it is well documented that miRNAs can target both oncogenes and tumor suppressor genes in a cell. The overall effect of a miRNA in a given cell results from its combined targeting effects on oncogenes and tumor suppressor genes [6]. Therefore, we need to fully understand what a specific miRNA (or ncRNA) does in a cell, in order to predict what to expect when we interfere with its expression for therapeutic reasons. To further complicate things, there is some evidence that the targeting effects of one miRNA on a gene, might be tissue- and/or species-specific (e.g., miR-29 family directly targets $D N M T 3 A$ and $3 B$ in human lung cancers [25], but it does not seem to target these genes in mouse embryonic stem cells [26]). Moreover, there is evidence of regulatory interactions between different groups of ncRNAs in cancer (e.g., the transcribed ultraconserved regions and miRNAs [27]), and the existence of possible feedback or feedforward regulatory loops should also be taken into consideration, because by 'therapeutically' modulating the expression of ncRNAs, we might end up modulating the expression of other ncRNAs as well, resulting in a synergistic or antagonistic effect. Fortunately, all these questions can be easily addressed preclinically, provided that more support is granted to encourage studies in this direction. Another variable that is part of the equation is the 'microenvironment' surrounding cancer cells. The discovery that miRNAs are secreted in exosome particles and can possibly transmit a 'message' from one cell to another, raises the question of the role of fibroblasts, and endothelial and inflammatory cells surrounding the tumors and of their role in carcinogenesis and invasiveness. The advent of laser singlecell dissection techniques will properly address these interactions and clarify which ncRNAs are involved, and by which cell they are released. Another point that needs to be highlighted is the surprising lack of interest from the ncRNA scientific community to the problem of 'dosage'. In other words, there seems to be no real consensus on the dose of a miRNA (or ncRNA) that should be administered to a cell in order to see a targeting effect. The literature reports huge differences in concentration ranges of miRNAs in transfection experiments, without considering (fortunately, not in all studies) the physiologic concentration of a miRNA, and the fact that what is seen experimentally as a direct 'target', might be just an artifact owing to the abnormally high (or low) doses of miRNA used in the experiment. The problem of the dosage will be claimed by translational research, and will have to be addressed before any clinical trial can truly be planned. Finally, in order to properly stop the metastasization process, we need to identify which ncRNAs (if any) are common to the different types of cancer in inducing metastases. A possible key to successfully address this issue is to promote 'meta-analysis' studies combining the already publicly available data on the several ncRNA expression-microarrays performed by different groups in almost all types of cancer. Combining these data seems to be the key to succeed in this intriguing challenge, which I would like to define as the next phase of ncRNA research. Overall, much work still needs to be done before we can claim that an efficient ncRNA-based anti-metastatic therapy has been developed; but the challenges are not impossible to overcome, especially considering that what is at stake is the very core of the malignant phenotype. 


\section{Financial \& competing interests disclosure}

The author has no relevant affiliations or financial involvement with any organization or entity with a financial interest in or financial conflict with the subject matter or materials discussed in the manuscript. This includes employment, consultancies, honoraria, stock ownership or options, expert testimony, grants or patents received or pending, or royalties.

No writing assistance was utilized in the production of this manuscript.

\section{Bibliography}

1 Weigelt B, Peterse JL, Van 'T Veer LJ: Breast cancer metastasis: markers and models. Nat. Rev. Cancer 5(8), 591-602 (2005).

2 Eccles SA, Welch DR: Metastasis: recent discoveries and novel treatment strategies. Lancet 369(9574), 1742-1757 (2007).

3 Fabbri M, Calin GA: Beyond genomics: interpreting the $93 \%$ of the human genome that does not encode proteins. Curr. Opin. Drug Discov. Devel. 13(3), 350-358 (2010).

4 Bartel DP: MicroRNAs: genomics, biogenesis, mechanism, and function. Cell 116(2), 281-297 (2004).

5 Calin GA, Sevignani C, Dumitru CD et al.: Human microRNA genes are frequently located at fragile sites and genomic regions involved in cancers. Proc. Natl Acad. Sci. USA 101(9), 2999-3004 (2004).

6 Fabbri M, Ivan M, Cimmino A, Negrini M, Calin GA: Regulatory mechanisms of microRNAs involvement in cancer. Expert Opin. Biol. Ther. 7(7), 1009-1019 (2007).

7 Garzon R, Fabbri M, Cimmino A, Calin GA, Croce CM: MicroRNA expression and function in cancer. Trends Mol. Med. 12(12), 580-587 (2006).
8 Fabbri M: miRNAs as molecular biomarkers of cancer. Expert Rev. Mol. Diagn. 10(4), 435-444 (2010).

9 Liu CG, Calin GA, Meloon B et al.: An oligonucleotide microchip for genome-wide microRNA profiling in human and mouse tissues. Proc. Natl Acad. Sci. USA 101(26), 9740-9744 (2004).

10 Lu J, Getz G, Miska EA et al.: MicroRNA expression profiles classify human cancers. Nature 435(7043), 834-838 (2005).

11 Chen C, Ridzon DA, Broomer AJ et al: Real-time quantification of microRNAs by stem-loop RT-PCR. Nucleic Acids Res. 33(20), E179 (2005).

12 Calin GA, Ferracin M, Cimmino A et al.: A microRNA signature associated with prognosis and progression in chronic lymphocytic leukemia. N. Engl. J. Med. 353(17), 1793-1801 (2005).

13 Calin GA, Croce CM: MicroRNA signatures in human cancers. Nat. Rev. Cancer 6(11), 857-866 (2006).

14 Fabbri M, Spizzo R, Calin GA: Highthroughput profiling in the hematopoietic system. Methods Mol. Biol. 667, 79-91 (2010).

15 Cimmino A, Calin GA, Fabbri $M$ et al. Mir-15 and mir-16 induce apoptosis by targeting bcl2. Proc. Natl Acad. Sci. USA 102(39), 13944-13949 (2005).

16 Croce CM: Causes and consequences of microRNA dysregulation in cancer. Nat. Rev. Genet. 10(10), 704-714 (2009).

17 Hou J, Wang P, Lin L et al.: MicroRNA-146a feedback inhibits RIG-I-dependent Type I IFN production in macrophages by targeting TRAF6, IRAK1, and IRAK2.J. Immunol. 183(3), 2150-2158 (2009).

18 Tavazoie SF, Alarcon C, Oskarsson T et al.: Endogenous human microRNAs that suppress breast cancer metastasis. Nature 451(7175), 147-152 (2008).
19 Hurst DR, Edmonds MD, Welch DR: Metastamir: the field of metastasis-regulatory microRNA is spreading. Cancer Res. 69(19), 7495-748 (2009).

20 Mitchell PS, Parkin RK, Kroh EM et al.: Circulating microRNAs as stable blood-based markers for cancer detection. Proc. Natl Acad. Sci. USA 105(30), 10513-10518 (2008).

21 Chen X, Ba Y, Ma L et al:: Characterization of microRNAs in serum: a novel class of biomarkers for diagnosis of cancer and other diseases. Cell. Res. 18(10), 997-1006 (2008).

22 Taylor DD, Gercel-Taylor C: MicrRNA signatures of tumor-derived exosomes as diagnostic biomarkers of ovarian cancer. Gynecol. Oncol. 110(1), 13-21 (2008).

23 Kan JS, Delassus GS, D’souza KG, Hoang S, Aurora R, Eliceiri Gl: Modulators of cancer cell invasiveness. J. Cell. Biochem. 111(4), 791-796 (2010).

24 Gupta RA, Shah N, Wang KC et al.: Long non-coding RNA hotair reprograms chromatin state to promote cancer metastasis. Nature 464(7291), 1071-1076 (2010).

25 Fabbri M, Garzon R, Cimmino A et al.: MicroRNA-29 family reverts aberrant methylation in lung cancer by targeting DNA methyltransferases $3 \mathrm{a}$ and $3 \mathrm{~b}$. Proc. Natl Acad. Sci. USA 104(40), 15805-15810 (2007).

26 Sinkkonen L, Hugenschmidt T, Berninger P et al:: MicroRNAs control de novo DNA methylation through regulation of transcriptional repressors in mouse embryonic stem cells. Nat. Struct. Mol. Biol. 15(3), 259-267 (2008).

27 Calin GA, Liu CG, Ferracin M et al.: Ultraconserved regions encoding ncRNAs are altered in human leukemias and carcinomas. Cancer Cell 12(3), 215-229 (2007). 